\title{
Combination of Erythromycin and Curcumin Alleviates Staphylococcus aureus Induced Osteomyelitis in Rats
}

\author{
Zubin Zhou ${ }^{\dagger}$, Chenhao Pan ${ }^{\dagger}$, Ye Lu, Youshui Gao, Wei Liu, Peipei Yin and Xiaowei Yu* \\ Department of Orthopaedic Surgery, Shanghai Jiao Tong University Affiliated Sixth People's Hospital, Shanghai, China
}

Osteomyelitis is commonly caused by Staphylococcus aureus. Both erythromycin and curcumin can suppress S. aureus growth, but their roles in osteomyelitis are barely studied. We aim to explore the activities of erythromycin and curcumin against chronical osteomyelitis induced by methicillin-resistant S. aureus (MRSA). Chronicle implant-induced osteomyelitis was established by MRSA infection in male Wistar rats. Four weeks after bacterial inoculation, rats received no treatment, erythromycin monotherapy, curcumin monotherapy, or erythromycin plus curcumin twice daily for 2 weeks. Bacterial levels, bone infection status, inflammatory signals and side effects were evaluated. Rats tolerated all treatments well, with no death or side effects such as, diarrhea and weight loss. Two days after treatment completion, erythromycin monotherapy did not suppress bacterial growth and had no effect in bone infection,

OPEN ACCESS

Edited by:

Margaret E. Bauer, Indiana University School of Medicine,

United States

Reviewed by:

Enrique Medina-Acosta, Universidade Estadual do Norte

Fluminense, Brazil Mathias Schmelcher,

ETH Zurich, Switzerland

*Correspondence: Xiaowei Yu

yuxw@sjtu.edu.cn

${ }^{\dagger}$ These authors have contributed equally to this work.

Received: 07 June 2017 Accepted: 09 August 2017

Published: 24 August 2017

Citation:

Zhou Z, Pan C, Lu Y, Gao Y, Liu W, Yin $P$ and $Y u X$ (2017) Combination of Erythromycin and Curcumin Alleviates Staphylococcus aureus Induced Osteomyelitis in Rats. Front. Cell. Infect. Microbiol. 7:379. doi: 10.3389/fcimb.2017.00379 although it reduced serum pro-inflammatory cytokines tumor necrosis factor (TNF)- $\alpha$ and interleukin (IL)-6. Curcumin monotherapy slightly suppressed bacterial growth, alleviated bone infection and reduced TNF- $\alpha$ and IL-6. Erythromycin and curcumin combined treatment markedly suppressed bacterial growth, substantially alleviated bone infection and reduced TNF- $\alpha$ and IL-6. Combination of erythromycin and curcumin lead a much stronger efficiency against MRSA induced osteomyelitis in rats than monotherapy. Our study suggests that erythromycin and curcumin could be a new combination for treating MRSA induced osteomyelitis.

Keywords: Staphylococcus aureus, osteomyelitis, erythromycin, curcumin, inflammation

\section{INTRODUCTION}

Staphylococcus aureus is the most common pathological microorganism that causes osteomyelitis (Lew and Waldvogel, 2004). Effective treatment for osteomyelitis is still lacking due to the formation of biofilm rendering it difficult for sufficient antibiotics to reach the local infection area as well as the development of resistance to antibiotic treatment. Although some drugs can temporarily suppress methicillin-resistant $S$. aureus (MRSA) induced osteomyelitis in animal models, severe side effects are observed, such as, diarrhea, malnutrition, and sometimes loss of research animals (Vergidis et al., 2015). Development of new safe and effective therapeutic strategies is highly demanded to overcome drug-resistant bacterial strains and adverse side effects. One such strategy is combination of different antibiotics or with plant derived natural products (Coutinho et al., 2009; Bezerra Dos Santos et al., 2015).

Erythromycin is broadly used to treat infections caused by various bacteria, including S. aureus (Jelic and Antolovic, 2016). The first use of erythromycin in clinical setting dated 
back to the early 1950s. The key mechanism for antibiotics of the macrolide family including erythromycin in suppressing bacterial growth is to inhibit the production of proteins crucial for bacterial function. In addition to direct targeting against bacteria, erythromycin also alleviates bacterial infection symptoms by suppressing inflammation (Cervin, 2001; Amsden, 2005). Erythromycin has been shown to reduce the production of pro-inflammatory cytokines in various scenarios (Miyajima et al., 1999; Desaki et al., 2004). While S. aureus frequently evolves to acquire resistance to antibiotic treatment, combination of erythromycin with other antibiotics or herbal medicines seems to be able to overcome this difficulty and results in a synergistic effect in suppressing bacterial growth (Bezerra Dos Santos et al., 2015). Interestingly, although the efficacy of erythromycin has been reported in various scenarios, its role in treating MRSA induced osteomyelitis is not well documented.

Some naturally occurring flavonoids have been shown to possess antibacterial functions against MRSA and can work synergistically with antibiotics such as, erythromycin to suppress bacterial growth (Chan et al., 2013). Curcumin, a major constituent of turmeric commonly used as a food spice, is a natural polyphenolic flavonoid isolated from the rhizome of the plant Curcuma longa Linné. Curcumin has been shown to exhibit various biological functions, including antioxidant, anti-inflammatory, anti-tumorigenic activities (Wang et al., 2009; Hussain et al., 2017; Zhao et al., 2017). It down-regulates pro-inflammatory signaling pathways, including transcription factors, cytokines, and other components that promote inflammation (Shehzad et al., 2013). It also possesses anti-microbial functions, including activity against $S$. aureus (Mishra et al., 2005; Rai et al., 2008; Tyagi et al., 2015). In addition, curcumin works synergistically with other antibiotics including oxacillin, ampicillin, ciprofloxacin, and norfloxacin against MRSA and significantly reduces the minimal inhibitory concentrations (MICs) of these antibiotics (Mun et al., 2013; Teow and Ali, 2015). Another in vitro study suggests that curcumin can reverse methicillin resistance in S. aureus (Mun et al., 2014). These observations lead us to hypothesize that curcumin may also be effective in suppressing MRSA growth in osteomyelitis.

In the current study, we explored the efficacies and side effects of erythromycin monotherapy, curcumin monotherapy, and erythromycin and curcumin combined therapy in a rat implant-induced osteomyelitis model caused by MRSA infection.

\section{MATERIALS AND METHODS}

\section{Bacteria}

S. aureus ATCC 43300 (a standard MRSA isolate; ATCC) was used to establish chronic osteomyelitis in rats.

\section{Rat Osteomyelitis Model}

All animal experiments were conducted in accordance with guidelines set by USA National Institutes of Health and standard protocols on materials with biosafety issue set by Shanghai Jiao Tong University Affiliated Sixth People's Hospital's Institutional Animal Care and Use Committee. This protocol of the animal study was also approved by Shanghai Jiao Tong University Affiliated Sixth People's Hospital's Institutional Animal Care and Use Committee (20160304). Adult male Wistar rats weighing $260 \mathrm{~g}$ to $340 \mathrm{~g}$ were purchased from SLAC Company and housed in Shanghai Jiao Tong University Affiliated Sixth People's Hospital rat facility under standard environment with free access to water and food. The procedures of establishing implant related osteomyelitis were adapted from previous studies (Vergidis et al., 2015; Guzel et al., 2016). Briefly, experimental rats were anesthetized by intraperitoneal injection of weight adjusted ketamine (60 mg/kg body weight) and xylazine $(6 \mathrm{mg} / \mathrm{kg}$ body weight). The left tibia was exposed after a small incision of the skin of the left hind limb. A hole was drilled in the bone using a $1 \mathrm{~mm}$ diameter titanium burr to allow access to medullary cavity. $10 \mu \mathrm{L}$ suspension containing $10^{8}$ colonyforming unit (CFU) of MRSA was injected into the medullary cavity of each exposed tibia. Following that, a $5 \mathrm{~mm}$ (length) $\times 1 \mathrm{~mm}$ (diameter) titanium Kirschner wire was inserted into the medullary cavity. Dental gypsum was used to cover the hole and the skin was sutured and treated with antiseptic solution. Subcutaneous administration of Buprenorphine was used for analgesia. Four weeks after surgery and bacteria inoculation, rats were randomly assigned to four groups, with 14 rats per group: control (no treatment), erythromycin monotherapy, curcumin monotherapy, and erythromycin plus curcumin. Both drugs were injected intraperitoneally every $12 \mathrm{~h}$ for 14 days. The dose for erythromycin (Sigma, MO, USA) is $20 \mathrm{mg} / \mathrm{kg}$ body weight and the dose for curcumin (Sigma, USA) is $50 \mathrm{mg} / \mathrm{kg}$ body weight. All rats were closely monitored for signs of adverse effects such as diarrhea and weight loss. $48 \mathrm{~h}$ after completion of drug treatment, rats from each study group were euthanized for MSRA culture and quantification, and histopathological evaluations.

\section{Microbiological Assessment}

Culture and quantification of bacteria from bone and wire were described previously (Vergidis et al., 2015). Briefly, the left tibia of each rat was aseptically removed. The bone were weighed and homogenized respectively. The homogenates were suspended in Trypticase soy broth, vortexed, diluted serially, plated onto sheep blood agar plates and cultured at $37^{\circ} \mathrm{C} .48 \mathrm{~h}$ after incubation, MRSA colonies were quantified and the results were expressed as $\log _{10} \mathrm{CFU} / \mathrm{g}$ of bone and $\log _{10} \mathrm{CFU} / \mathrm{cm}^{2}$ of wire surface.

\section{Histopathological Assessment}

$48 \mathrm{~h}$ after treatment, tibia from rats of each treatment group were fixed in formaldehyde and sectioned into $5 \mu \mathrm{m}$ thick longitudinal sagittal sections. Bone slices were stained with hematoxylin and eosin (H\&E) using standard procedures as described previously (Fischer et al., 2008). Four regions of interest (ROI) were assessed for bone infection, including proximal epi-/metaphysis and diaphysis, and distal epi-/metaphysis and diaphysis. Each ROI was assessed by four independent observers, with no access to the animal group codes. Scores were assigned based on the criteria as described in previous studies (Lucke et al., 2003; Guzel et al., 2016). The following four parameters were scored with 0 (absent) or 1 (present): 1. Abscess formation; 2. Sequestrum formation; 3. Enlargement of corticalis; 4. Destruction of 
corticalis. The remaining 5th parameter (general impression) was scored as 0 (absent), 1 (mild), or 2 (severe). The maximum score of one tibia including four ROIs and five parameters is 24 .

\section{Elisa}

Levels of tumor necrosis factor-alpha (TNF- $\alpha$ ) and interleukin-6 (IL-6) in rat serum were detected by rat enzyme-linked immunosorbent assay (ELISA) kits (both purchased from R\&D Biosystem, Minneapolis, MN, USA) according to manufacturer's instruction. Results were represented as $\mathrm{pg} / \mathrm{mL}$ of serum. Serum from rats of each group was collected $48 \mathrm{~h}$ after treatment.

\section{Statistical Analysis}

Data were analyzed by SPSS 21.0 (SPSS Inc., Chicago, IL, USA) and presented as mean $\pm S D$. The difference between two groups was determined by one-way ANOVA analysis followed by a Tukey's post hoc test. The difference was regarded statistical significant when $p$ value $<0.05$.

\section{RESULTS}

\section{Adverse Effects of Erythromycin and/or Curcumin Treatments in Rats with Osteomyelitis}

We closely monitored all the rats undergoing different treatments, including those in the control group. We found that the rats tolerated all the treatments well and all rats survived the whole study duration. No rats developed diarrhea or weight loss. In fact we observed a significant increase of body weight from 2 weeks post treatment when rats were treated with erythromycin alone or a combination of erythromycin and curcumin, compared to that of control group (Figure 1). Curcumin treatment alone did not significantly alter rat body weight.

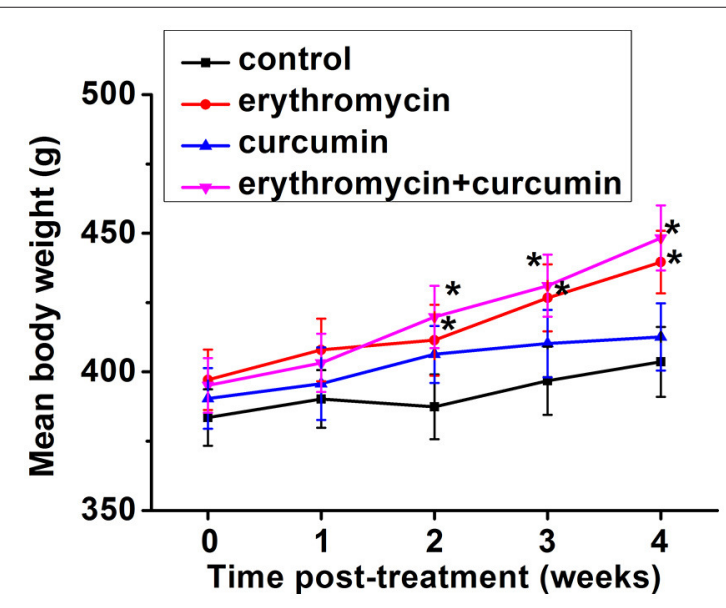

FIGURE 1 | Mean body weight in each experimental group at indicated time points post treatment. Results were presented as mean $\pm S D{ }^{*} p<0.05$ vs. control.

\section{Combination of Erythromycin and Curcumin Strongly Suppressed MRSA Growth}

We evaluated bacterial growth in rats from all four groups $48 \mathrm{~h}$ after treatment. We found that treatment of rats with erythromycin alone had no effect on bacterial levels compared to that of control rat. Curcumin treatment alone slightly but significantly suppressed bacterial growth. Erythromycin and curcumin double treatment drastically reduced bacterial levels both in the bone (Figure 2A) and wire (Figure 2B). These results suggest that erythromycin and curcumin had a strong effect on suppressing MRSA growth.

\section{Reduced Bone Infection as a Result of Erythromycin and Curcumin Double Treatment}

Forty-eight hour after treatment of rats with osteomyelitis, we examined bone tissue lesions using $\mathrm{H} \& \mathrm{E}$ staining (Figure 3A). We found that control rats showed severe signs of infection with a high average histopathological score (Figures 3A, B). Erythromycin treatment alone did not alleviate the status of osteomyelitis and the histopathological score was similar to that of control rats. Curcumin treatment alone slightly reduced bone infection with a significantly lower histopathological score. Importantly, combination of erythromycin and curcumin substantially suppressed bone lesions and decreased the histopathological score.

\section{Reduced Pro-Inflammatory Cytokine Levels in Erythromycin and Curcumin Double Treated Rats}

We examined levels of two pro-inflammatory cytokines TNF- $\alpha$ and IL- 6 by ELISA. We found that all three treatments (erythromycin monotherapy, curcumin monotherapy, and erythromycin plus curcumin) significantly reduced the levels of TNF- $\alpha$ (Figure 4A) and IL-6 (Figure 4B), with the lowest levels observed in the group of erythromycin plus curcumin treatment. These results suggest that erythromycin and curcumin treatment greatly reduced inflammation induced by osteomyelitis in rats.

\section{DISCUSSION}

Implant-induced osteomyelitis is commonly caused by S. aureus. Effective treatment is still lacking due to formation of biofilm making drug difficult to access as well as the generation of antibiotic resistant bacterial strains. Rat models enable us to study new ways to treat osteomyelitis.

In the current study, we successfully established chronic implant-induced osteomyelitis in male Wistar rats as described previously (Lucke et al., 2003; Vergidis et al., 2015; Guzel et al., 2016). Using this model, we studied the effects of erythromycin alone, curcumin alone and erythromycin and curcumin combined treatment against MRSA induced osteomyelitis. We found that although erythromycin or curcumin monotherapy did not show very effective alleviation of bone infection, combined treatment of these two drugs remarkably suppressed 


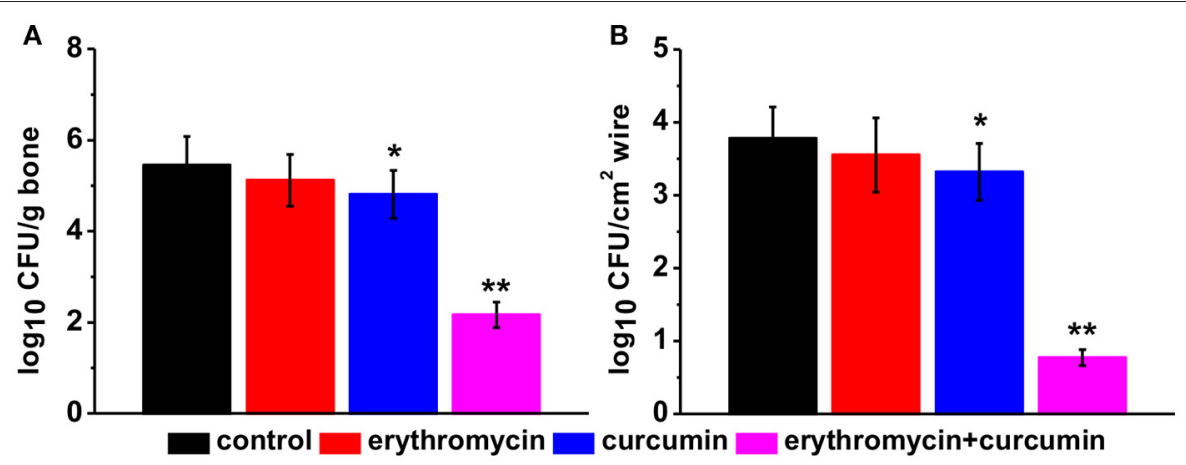

FIGURE 2 | Bacterial levels in each experimental group $48 \mathrm{~h}$ post treatment, quantified in bone (A) and wire (B) cultures expressed as log 10 colony forming units (CFU) per tissue $(\mathrm{g})$ and per wire surface $\left(\mathrm{cm}^{2}\right)$, respectively. Results were presented as mean $\pm S D{ }^{*} p<0.05$ and ${ }^{* *} p<0.01$ vs. control.

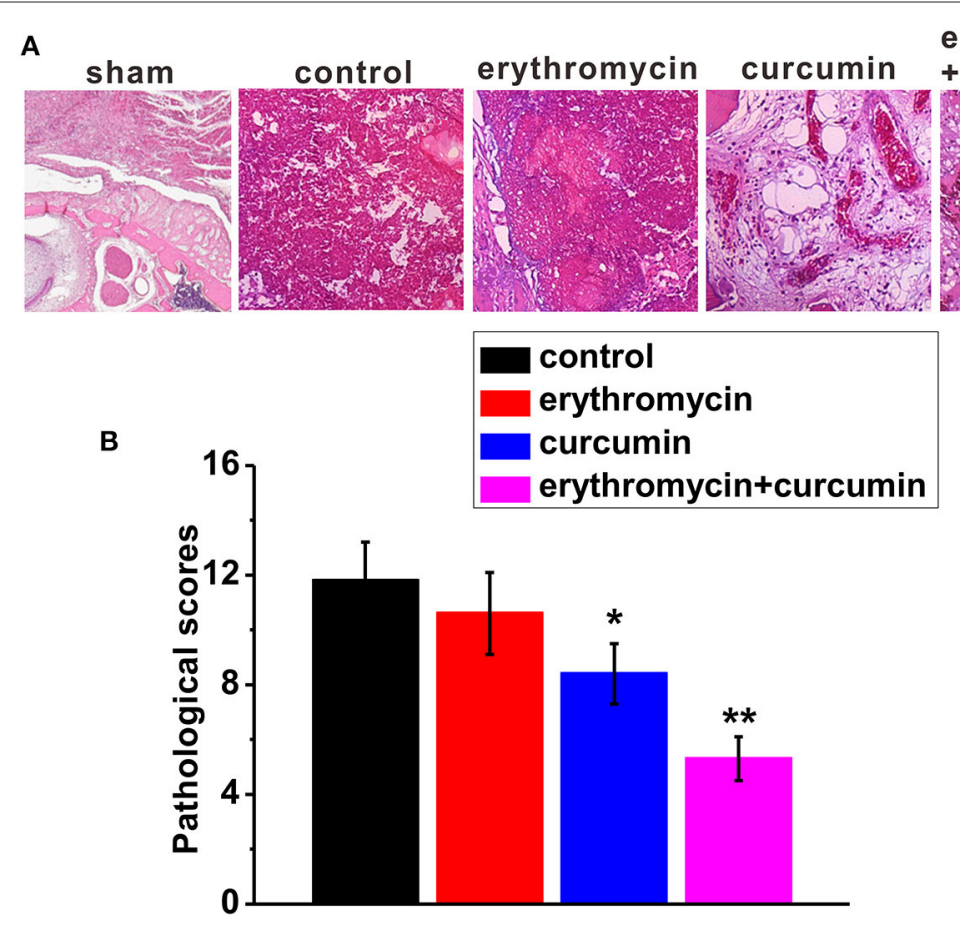

FIGURE 3 | Histological examination in the tibiae of experimental rats. Representative images of H\&E staining (A) and histological scores (B) in each experimental group. Note that the representative image of sham was from a healthy bone. Results were presented as mean $\pm S D{ }^{*} p<0.05$ and ${ }^{* *} p<0.01$ vs. control.

bacterial growth, alleviated bone infection status and reduced pro-inflammatory cytokines TNF- $\alpha$ and IL-6. To our best knowledge, this is the first study reporting a significant beneficial effect of erythromycin and curcumin against MRSA infection and osteomyelitis in rats.

Based on its antimicrobial function against $S$. aureus, we originally hypothesized that erythromycin would suppress bacterial growth in our rat model of osteomyelitis that is caused by MRSA infection. Surprisingly, we found that the bacteria levels in the bone gone through 2 weeks of erythromycin treatment did not significantly differ from that of control group. The histopathological status was also similar to control rats. This may be due to resistance of the bacteria toward antibiotics. Minimum inhibitory concentration (MIC) of erythromycin against MRSA in this study was $2.3 \mathrm{mg} / \mathrm{L}$. Thus, MRSA should be resistant to erythromycin according to current literature (Westh et al., 1991). Evolvement of bacteria resistance to antibiotics is a big obstacle for antibiotics in treating S. aureus induced infections. This frequently renders antibiotic monotherapy inefficient. In fact, it has been shown previously that addition of erythromycin in cultured mouse osteoblasts at the time of $S$. aureus infection prevented bacteria growth while treatment at $12 \mathrm{~h}$ after infection had no such effect (Ellington et al., 2006). 

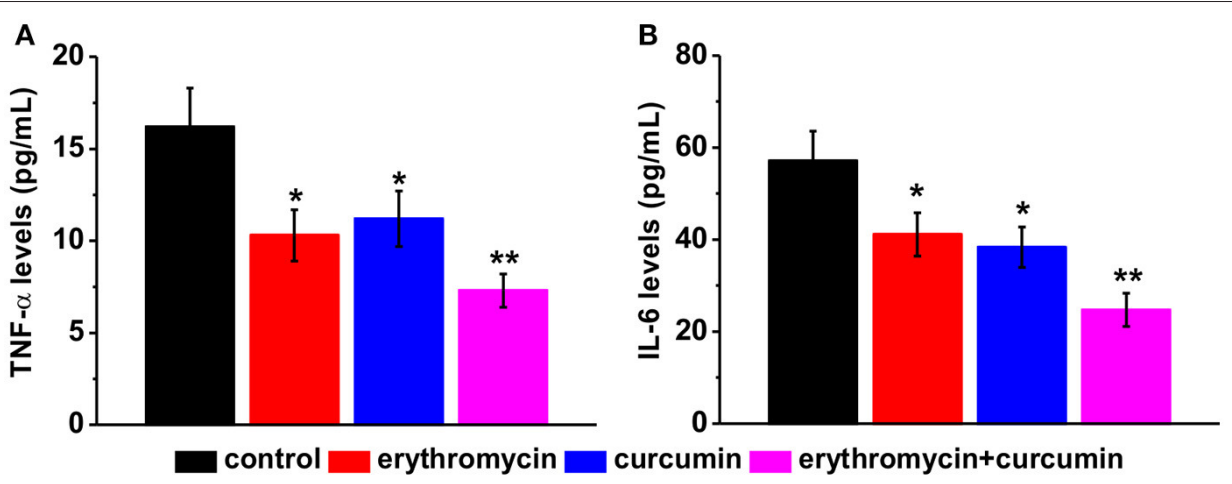

FIGURE 4 | Levels of pro-inflammatory cytokines in the serum of experimental rats after treatment, including TNF- $\alpha$ (A) and IL-6 (B), measured by ELISA. Results were presented as mean $\pm S D{ }^{*} p<0.05$ and ${ }^{* *} p<0.01$ vs. control.

One approach to overcome bacterial resistance is by combining different antibiotics or using herbal medicines together with antibiotics (Allen and Epp, 1978; Hemaiswarya et al., 2008). Some plant-derived products have shown synergistic effect when combining with erythromycin against bacterial infection. For example, organic extracts from Indigofera suffruticosa leaves and erythromycin synergistically suppress S. aureus (Bezerra Dos Santos et al., 2015).

Curcumin, a naturally occurring flavonoid, exerts an antibacterial activity against $S$. aureus. It was not clear whether curcumin has any beneficial effect in treating MRSA induced osteomyelitis. In the current study, we investigated this possibility and found only a slight antibacterial effect when treated alone in rats with chronic implant-induced osteomyelitis. Similar to the synergism of curcumin and other antibiotics against $S$. aureus, we also observed a remarkable effect of curcumin and erythromycin combined treatment on suppressing bacteria growth.

Inflammation is associated with bacterial infection. Previous studies showed that increased inflammation is associated with osteomyelitis (Yoshii et al., 2002; Lew and Waldvogel, 2004; Guzel et al., 2016). Despite of its lacking antimicrobial function in MRSA induced osteomyelitis, we did observe a significant reduction of pro-inflammatory cytokines in rats treated with erythromycin alone. This is consistent with previous studies showing that erythromycin can inhibit cytokine gene expressing including TNF- $\alpha$ and IL-6 (Schultz et al., 1998; Cervin, 2001). Curcumin alone or combined with erythromycin also reduced TNF- $\alpha$ and IL-6, with combined treatment showing lower levels of TNF- $\alpha$ and IL- 6 compared to curcumin or erythromycin alone. It is worth noting that osteomyelitis model was induced by injection of suspensions of MRSA cell in the current study, which might introduce systemic bacterial infection. Other methods to deliver the MRSA cells locally into the bone, for example the use of agarose beads containing MRSA (Marriott et al., 2005), may reduce the risk of systemic infection. This is one of the limitations of our study.

In addition to anti-microbial and anti-inflammatory efficiencies, development of adverse side effects is a big concern for determination of the ideal treatment. Severe adverse effects could result from antibiotic treatment, including diarrhea, vomiting, malnutrition, and weight loss (Vergidis et al., 2015). We checked our experimental rats closely for side effects resulted from MRSA infection and drug treatment. All the rats tolerated our treatment regimens well, with no rats developing diarrhea or weight loss. In fact, we noticed an increase of body weight when rats were treated with erythromycin or a combination of erythromycin and curcumin. Curcumin treatment alone did not change body weight compared to that of control rats.

Future directions will include determination of potential bacterial resistance, MICs, long term effects, and local application of the drugs. Further studies are needed to determine if combination of erythromycin and curcumin indeed is an ideal therapeutic strategy for treating implant-induced osteomyelitis in human patients.

\section{CONCLUSION}

We demonstrated here that combination of erythromycin and curcumin has a much stronger antibacterial effect against MRSA in a rat model of implant-induced osteomyelitis than each individual therapy. This treatment was well tolerated, with no death outcome during the experimental period. Our study gives a potential new therapeutic direction for $S$. aureus induced infections and inflammation.

\section{AUTHOR CONTRIBUTIONS}

Did the experiments and analyzed the data: ZZ, CP, YL, YG, WL, PY. Designed the study and wrote the manuscript: XY. All authors approved the final submission.

\section{FUNDING}

This work was supported by the National Natural Science Foundation of China (No. 81472110, 81572155), Shanghai Municipal Natural Science Foundation (No. 14ZR1431800). 


\section{REFERENCES}

Allen, N. E., and Epp, J. K. (1978). Mechanism of penicillin-erythromycin synergy on antibiotic-resistant Staphylococcus aureus. Antimicrob. Agents Chemother. 13, 849-853. doi: 10.1128/AAC.13.5.849

Amsden, G. W. (2005). Anti-inflammatory effects of macrolides-an underappreciated benefit in the treatment of community-acquired respiratory tract infections and chronic inflammatory pulmonary conditions? J. Antimicrob. Chemother. 55, 10-21. doi: 10.1093/jac/dkh519

Bezerra Dos Santos, A. T., Araujo, T. F., Nascimento Da Silva, L. C., Da Silva, C. B., De Oliveira, A. F., Araujo, J. M., et al. (2015). Organic extracts from Indigofera suffruticosa leaves have antimicrobial and synergic actions with erythromycin against Staphylococcus aureus. Front. Microbiol. 6:13. doi: $10.3389 /$ fmicb. 2015.00013

Cervin, A. (2001). The anti-inflammatory effect of erythromycin and its derivatives, with special reference to nasal polyposis and chronic sinusitis. Acta Otolaryngol. 121, 83-92. doi: 10.1080/0001648013000 06326

Chan, B. C., Ip, M., Gong, H., Lui, S. L., See, R. H., Jolivalt, C., et al. (2013). Synergistic effects of diosmetin with erythromycin against $A B C$ transporter over-expressed methicillin-resistant Staphylococcus aureus (MRSA) RN4220/pUL5054 and inhibition of MRSA pyruvate kinase. Phytomedicine 20, 611-614. doi: 10.1016/j.phymed.2013.02.007

Coutinho, H. D., Costa, J. G., Lima, E. O., Falcao-Silva, V. S., and Siqueira, J. P. Jr. (2009). Herbal therapy associated with antibiotic therapy: potentiation of the antibiotic activity against methicillin-resistant Staphylococcus aureus by Turnera ulmifolia L. BMC Complement. Altern. Med. 9:13. doi: 10.1186/1472-6882-9-13

Desaki, M., Okazaki, H., Sunazuka, T., Omura, S., Yamamoto, K., and Takizawa, H. (2004). Molecular mechanisms of anti-inflammatory action of erythromycin in human bronchial epithelial cells: possible role in the signaling pathway that regulates nuclear factor- $\mathrm{B}$ activation. Antimicrob. Agents Chemother. 48, 1581-1585. doi: 10.1128/AAC.48.5.1581-1585.2004

Ellington, J. K., Harris, M., Hudson, M. C., Vishin, S., Webb, L. X., and Sherertz, R. (2006). Intracellular Staphylococcus aureus and antibiotic resistance: implications for treatment of staphylococcal osteomyelitis. J. Orthop. Res. 24, 87-93. doi: 10.1002/jor.20003

Fischer, A. H., Jacobson, K. A., Rose, J., and Zeller, R. (2008). Hematoxylin and eosin staining of tissue and cell sections. CSH Protoc. 2008:prot4986. doi: $10.1101 /$ pdb.prot 4986

Guzel, Y., Golge, U. H., Goksel, F., Vural, A., Akcay, M., Elmas, S., et al. (2016). The efficacy of boric acid used to treat experimental Osteomyelitis caused by methicillin-resistant Staphylococcus aureus: an in vivo study. Biol. Trace Elem. Res. 173, 384-389. doi: 10.1007/s12011-016-0662-y

Hemaiswarya, S., Kruthiventi, A. K., and Doble, M. (2008). Synergism between natural products and antibiotics against infectious diseases. Phytomedicine 15, 639-652. doi: 10.1016/j.phymed.2008.06.008

Hussain, Z., Thu, H. E., Amjad, M. W., Hussain, F., Ahmed, T. A., and Khan, S. (2017). Exploring recent developments to improve antioxidant, antiinflammatory and antimicrobial efficacy of curcumin: a review of new trends and future perspectives. Mater. Sci. Eng. C Mater. Biol. Appl. 77, 1316-1326. doi: 10.1016/j.msec.2017.03.226

Jelic, D., and Antolovic, R. (2016). From erythromycin to azithromycin and new potential ribosome-binding antimicrobials. Antibiotics 5:E29. doi: 10.3390/antibiotics5030029

Lew, D. P., and Waldvogel, F. A. (2004). Osteomyelitis. Lancet 364, 369-379. doi: 10.1016/S0140-6736(04)16727-5

Lucke, M., Schmidmaier, G., Sadoni, S., Wildemann, B., Schiller, R., Stemberger, A., et al. (2003). A new model of implant-related osteomyelitis in rats. J. Biomed. Mater. Res. Part B Appl. Biomater. 67, 593-602. doi: 10.1002/jbm.b.10051

Marriott, I., Gray, D. L., Rati, D. M., Fowler, V. G. Jr., Stryjewski, M. E., Levin, L. S., et al. (2005). Osteoblasts produce monocyte chemoattractant protein-1 in a murine model of Staphylococcus aureus osteomyelitis and infected human bone tissue. Bone 37, 504-512. doi: 10.1016/j.bone.2005.05.011

Mishra, S., Narain, U., Mishra, R., and Misra, K. (2005). Design, development and synthesis of mixed bioconjugates of piperic acid-glycine, curcumin-glycine/alanine and curcumin-glycine-piperic acid and their antibacterial and antifungal properties. Bioorg. Med. Chem. 13, 1477-1486. doi: 10.1016/j.bmc.2004.12.057

Miyajima, M., Suga, M., Nakagawa, K., Ito, K., and Ando, M. (1999). Effects of erythromycin on experimental extrinsic allergic alveolitis. Clin. Exp. Allergy 29, 253-261. doi: 10.1046/j.1365-2222.1999.00430.x

Mun, S. H., Joung, D. K., Kim, Y. S., Kang, O. H., Kim, S. B., Seo, Y. S., et al. (2013). Synergistic antibacterial effect of curcumin against methicillin-resistant Staphylococcus aureus. Phytomedicine 20, 714-718. doi: 10.1016/j.phymed.2013.02.006

Mun, S. H., Kim, S. B., Kong, R., Choi, J. G., Kim, Y. C., Shin, D. W., et al. (2014). Curcumin reverse methicillin resistance in Staphylococcus aureus. Molecules 19, 18283-18295. doi: 10.3390/molecules191118283

Rai, D., Singh, J. K., Roy, N., and Panda, D. (2008). Curcumin inhibits FtsZ assembly: an attractive mechanism for its antibacterial activity. Biochem. J. 410, 147-155. doi: 10.1042/BJ20070891

Schultz, M. J., Speelman, P., Zaat, S., Van Deventer, S. J., and Van Der Poll, T. (1998). Erythromycin inhibits tumor necrosis factor alpha and interleukin 6 production induced by heat-killed Streptococcus pneumoniae in whole blood. Antimicrob. Agents Chemother. 42, 1605-1609.

Shehzad, A., Rehman, G., and Lee, Y. S. (2013). Curcumin in inflammatory diseases. Biofactors 39, 69-77. doi: 10.1002/biof.1066

Teow, S. Y., and Ali, S. A. (2015). Synergistic antibacterial activity of Curcumin with antibiotics against Staphylococcus aureus. Pak. J. Pharm. Sci. 28, 2109-2114.

Tyagi, P., Singh, M., Kumari, H., Kumari, A., and Mukhopadhyay, K. (2015). Bactericidal activity of curcumin I is associated with damaging of bacterial membrane. PLoS ONE 10:e0121313. doi: 10.1371/journal.pone.0121313

Vergidis, P., Schmidt-Malan, S. M., Mandrekar, J. N., Steckelberg, J. M., and Patel, R. (2015). Comparative activities of vancomycin, tigecycline and rifampin in a rat model of methicillin-resistant Staphylococcus aureus osteomyelitis. J. Infect. 70, 609-615. doi: 10.1016/j.jinf.2014.12.016

Wang, Y., Lu, Z., Wu, H., and Lv, F. (2009). Study on the antibiotic activity of microcapsule curcumin against foodborne pathogens. Int. J. Food Microbiol. 136, 71-74. doi: 10.1016/j.ijfoodmicro.2009.09.001

Westh, H., Knudsen, A. M., Gottschau, A., and Rosdahl, V. T. (1991). Evolution of Staphylococcus aureus resistance to erythromycin in Denmark, 1959 to 1988: correlations between characteristics of erythromycin-resistant bacteraemia strains. J. Hosp. Infect. 18, 35-43. doi: 10.1016/0195-6701(91)90091-L

Yoshii, T., Magara, S., Miyai, D., Nishimura, H., Kuroki, E., Furudoi, S., et al. (2002). Local levels of interleukin-1 $\beta,-4,-6$ and tumor necrosis factor $\alpha$ in an experimental model of murine osteomyelitis due to Staphylococcus aureus. Cytokine 19, 59-65. doi: 10.1006/cyto.2002.1039

Zhao, S., Ma, L., Cao, C., Yu, Q., Chen, L., and Liu, J. (2017). Curcuminloaded redox response of self-assembled micelles for enhanced antitumor and anti-inflammation efficacy. Int. J. Nanomed. 12, 2489-2504. doi: $10.2147 /$ IJN.S123190

Conflict of Interest Statement: The authors declare that the research was conducted in the absence of any commercial or financial relationships that could be construed as a potential conflict of interest.

Copyright $\odot 2017$ Zhou, Pan, Lu, Gao, Liu, Yin and Yu. This is an open-access article distributed under the terms of the Creative Commons Attribution License (CC BY). The use, distribution or reproduction in other forums is permitted, provided the original author(s) or licensor are credited and that the original publication in this journal is cited, in accordance with accepted academic practice. No use, distribution or reproduction is permitted which does not comply with these terms. 